\title{
A rare case of renal cell carcinoma with hematogenous extension into the right atrium discovered incidentally on echocardiogram
}

\author{
Ryan Dean BS, MBA, Ganesh Maniam BA, MBA, Thien Vo MD
}

\begin{abstract}
While hematogenous spread of renal cell carcinoma (RCC) is common, isolated extension into the renal vein and inferior vena cava (IVC) is rare, and extension into the right atrium is even less likely. This 62-year-old Hispanic woman was admitted for a suspected inferior myocardial infarction; her echocardiogram revealed a right atrial mass consistent with an atrial myxoma. The atrial mass was excised during coronary artery bypass surgery, and the histology was consistent with metastatic clear cell RCC. Computed tomography of the abdomen demonstrated a $5 \mathrm{~cm}$ right lower pole renal mass with hilar involvement and filling defects in the IVC and the right renal vein; these findings were consistent with RCC tumor thrombus extension into the renal vein, IVC, and right atrium. The radical nephrectomy necessary for tumor removal could not be performed at this hospital, and the patient was discharged to a higher level of care. The incidence of RCC with extension into the right atrium is quite low, but clinicians should understand that the lethality of RCC warrants immediate clinical investigation upon diagnosis.
\end{abstract}

Keywords: Clear cell renal carcinoma, echocardiography, right atrium, metastasis, incidental findings

\section{INTRODUCTION}

Renal cell carcinoma $(\mathrm{RCC})$ is considered the most lethal urological malignancy, since $30 \%$ of patients initially diagnosed already have metastatic disease after staging. ${ }^{1}$ However, the increased availability and use of imaging modalities have increased the detection of these renal neoplasms, such that over $70 \%$ of these cases are detected incidentally. ${ }^{2}$ While hematogenous spread of RCC is common, isolated extension into the renal vein and inferior vena cava (IVC) is rare and extension to the right atrium is even less likely. ${ }^{2}$ This paper presents a rare case of an RCC with hematogenous extension into the right atrium via the IVC, presenting incidentally as a right atrial mass

Corresponding author: Ganesh Maniam Contact Information: Ganesh.maniam@ttuhsc.edu DOI: 10.12746/swrccc.v8i34.655 on an echocardiogram performed for evaluation of an inferior wall myocardial infarction.

\section{CASE REPORT}

A 62-year-old Hispanic woman was admitted for a suspected inferior myocardial infarction, and her echocardiogram revealed a right atrial mass consistent with an atrial myxoma (Figure 1). The patient had presented with chest pain but did not have dyspnea or syncope. On the fourth day of hospitalization, the patient underwent coronary artery bypass surgery (greater saphenous vein harvest from left leg) for triple-vessel disease with left main involvement and excision of the atrial mass. Histopathological examination of the mass revealed a clear cell tumor positive for EGFR, PAXB, and CD10, consistent with metastatic clear cell RCC. The following day a renal ultrasound was performed but was inconclusive. Abdominal computed tomography (CT) demonstrated 


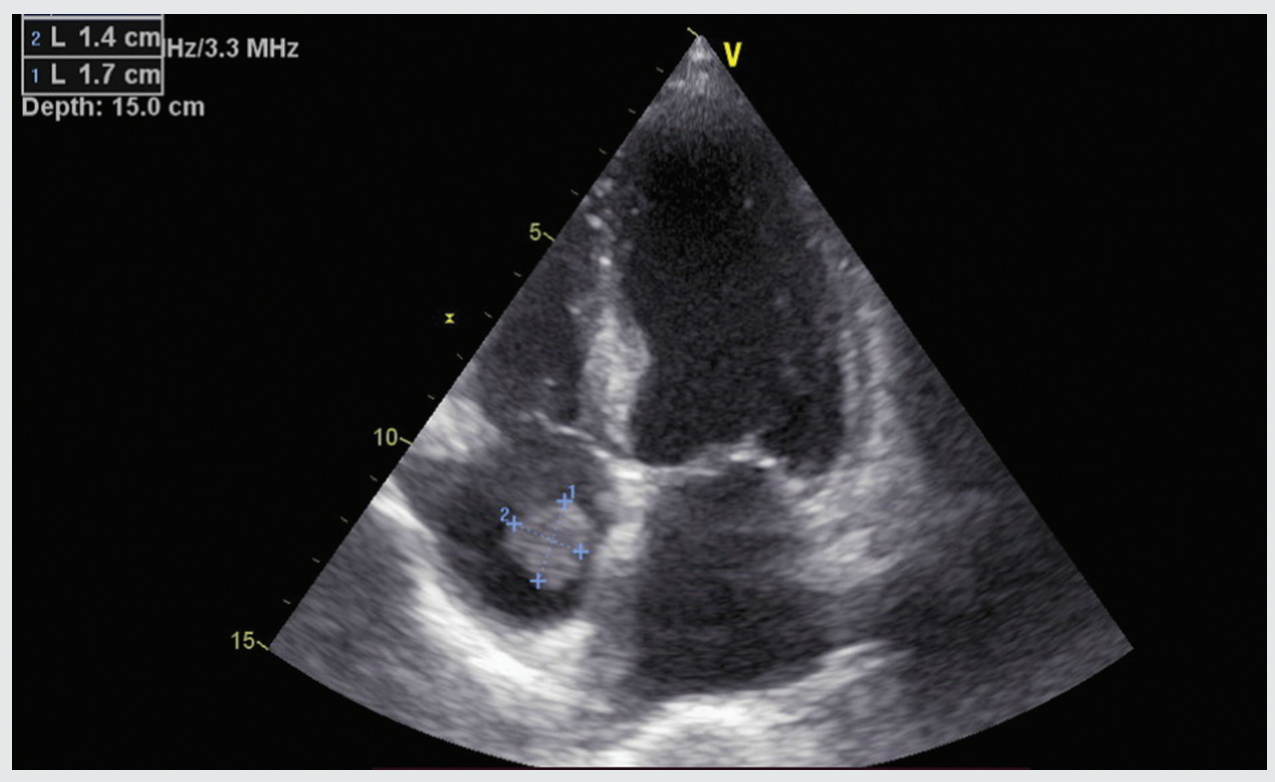

Figure 1. Echocardiogram performed for suspected inferior myocardial infarction, which had also revealed a right atrial mass.

a $5 \mathrm{~cm}$ right lower pole renal mass with hilar involvement and filling defects in the IVC and the right renal vein (Figures 2-5); these findings were consistent with RCC tumor thrombus extension into the renal vein, IVC, and right atrium. Computed tomography of the thorax and head found no evidence of lung or brain metastasis. The diagnosis and prognosis were discussed with the patient, including the recommendation for radical nephrectomy for RCC and IVC tumor removal. This surgery could not be performed at this hospital, and she was referred to another hospital for management. The patient has been lost to follow-up.

\section{Discussion}

The rate of initial presentation with metastatic RCC is likely decreasing due to increased utilization of imaging modalities, thereby increasing the rate of incidental diagnosis, so that more cases are diagnosed before metastasis has occurred. ${ }^{2}$ Metastasis of RCC commonly involves the lungs, brain, and intra-abdominal organs; metastasis to the cardiovascular system, specifically the renal vein and IVC, is much less commonapproximately $10 \%$ of RCC patients-but is associated with a relatively good prognosis following surgical excision. ${ }^{2}$ Metastasis to the heart, typically the right atrium via the IVC, occurs in less than $1 \%$ of RCC patients. ${ }^{2}$

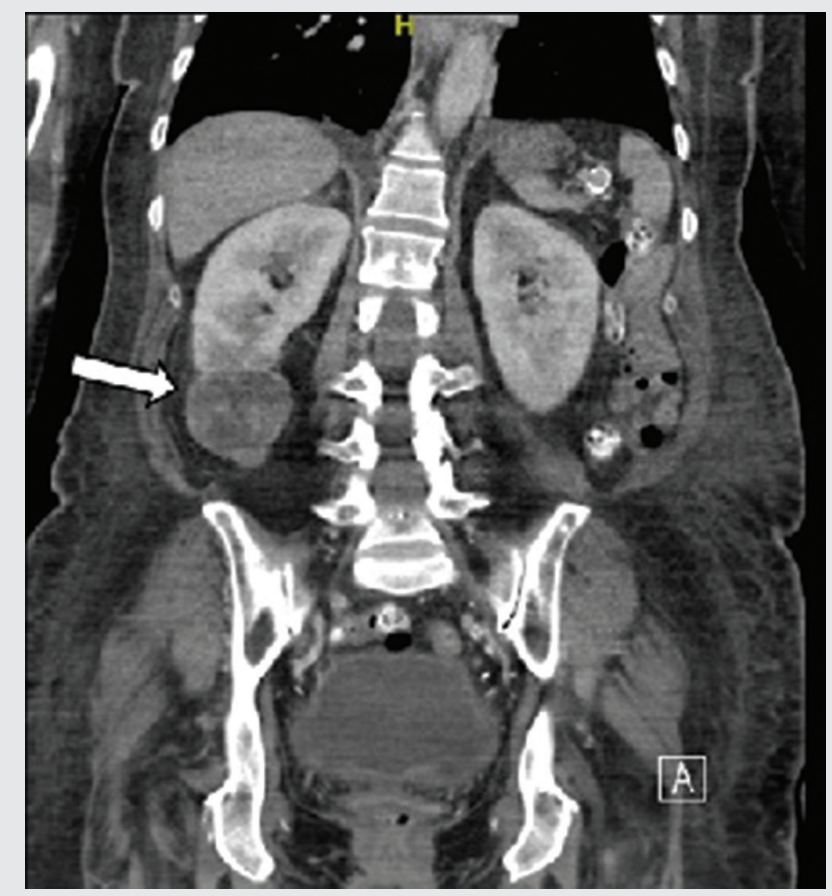

Figure 2. Abdominal CT in coronal view demonstrating mass on right kidney lower pole (arrow); lesions demonstrates increased density on the pre-contrast suggestive of blood products. 


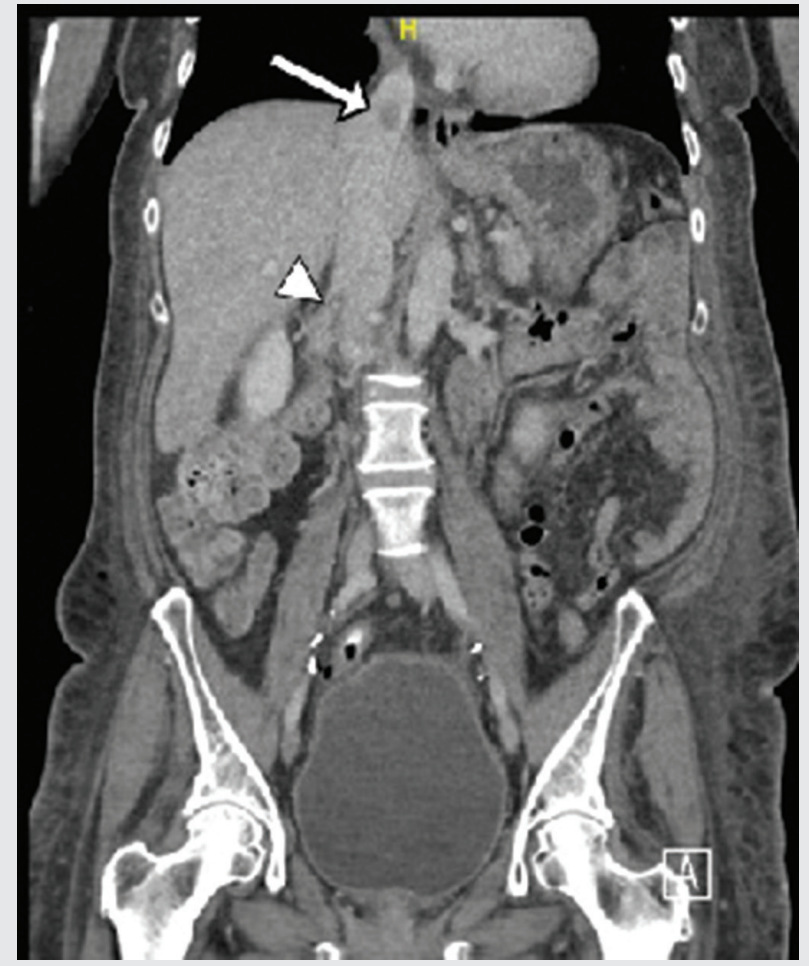

Figure 3. Abdominal $\mathrm{CT}$ in coronal view demonstrating filling defects in the IVC (arrow) and infrahepatic IVC with extension into the right renal vein (arrowhead) consistent with tumor thrombus.

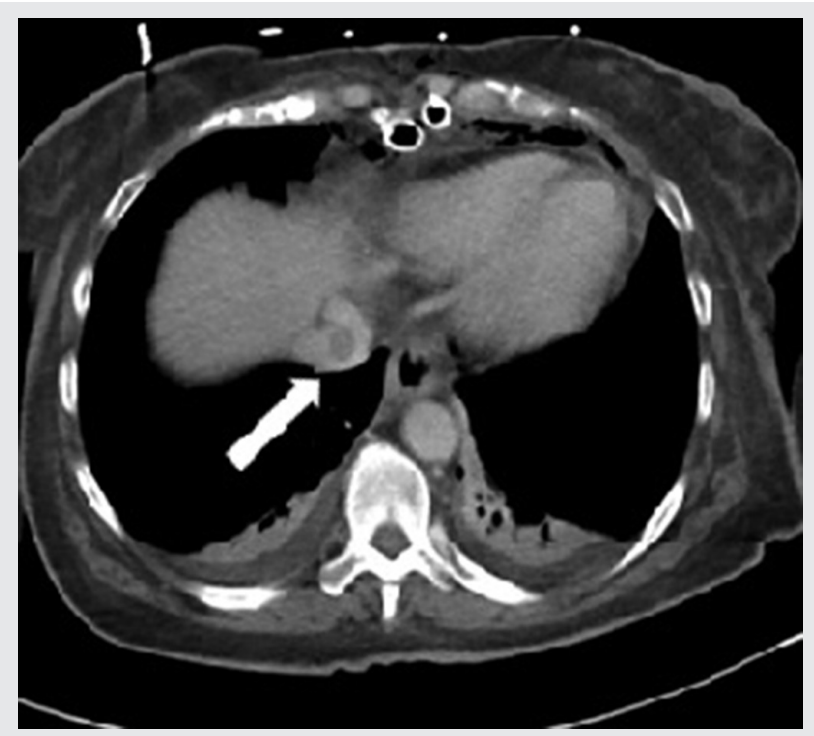

Figure 4. Abdominal $\mathrm{CT}$ with contrast in axial view demonstrating filling defects in the IVC (arrow).

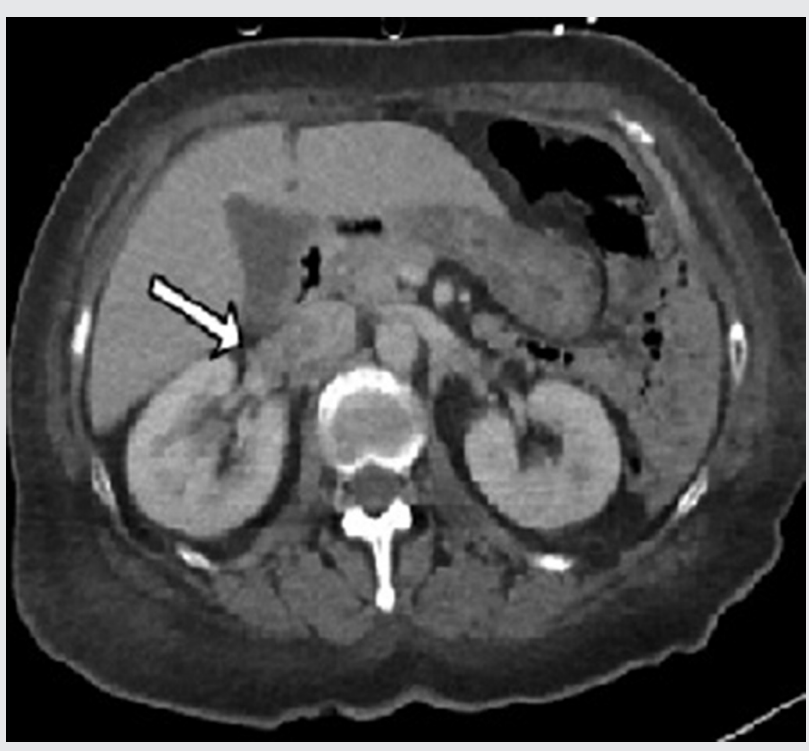

Figure 5. Abdominal $\mathrm{CT}$ with contrast in axial view demonstrating filling defects in the infrahepatic IVC with extension into the right renal vein (arrow) consistent with tumor thrombus.

A review of case reports between 2006 and 2011 found only 10 reported cases of cardiac metastasis of RCC. ${ }^{2}$ Hence, RCC invasion of the right atrium is exceedingly rare, and other reports in the literature are sparse. One early case series of 18 patients between 1984-1993 reported a 5-year survival rate of $60.2 \%{ }^{3}$ Another case presented a 59-year-old woman with RCC protruding from the IVC into the right atrium, which the authors state has a significantly worse prognosis than other levels of IVC involvement. ${ }^{4}$ Additionally, there is a unique case report of RCC metastasis into the right atrium without involvement of the IVC. ${ }^{5}$ Finally, a published case describes Budd-Chiari syndrome secondary to RCC extension of the IVC and right atrium. ${ }^{6}$

This paper presents a rare case of RCC extension into the right atrium via hematogenous metastasis through the renal vein and IVC, which presented incidentally as a right atrial mass on an echocardiogram performed for an inferior wall myocardial infarction. Clinicians should understand that such cases are certainly rare, but that the lethality of RCC warrants immediate clinical investigation and management upon diagnosis. In this case, the patient was recommended 
for a radical nephrectomy for RCC and IVC tumor removal, which is associated with a relatively good prognosis based on previous case reports; neither of these procedures could be performed at this hospital, and she required transfer to a higher level of care at a different facility. The increased utilization of sophisticated imaging modalities will likely continue to increase the rate of incidental discovery of such neoplasms, and physicians should keep RCC in the differential diagnosis when a right atrial mass is discovered incidentally on an echocardiogram.

Article citation: Dean R, Maniam G, Vo T. A rare case of renal cell carcinoma with hematogenous extension into the right atrium discovered incidentally on echocardiogram. The Southwest Respiratory and Critical Care Chronicles 2020;8(34):52-55

From: Department of Internal Medicine, Texas Tech University Health Sciences Center, Amarillo, Texas

Submitted: $2 / 23 / 2020$

Accepted: 4/19/2020

Reviewer: Sanket Thakore MD

Conflicts of interest: none

This work is licensed under a Creative Commons Attribution-ShareAlike 4.0 International License.

\section{REFERENCES}

1. Huang Q, Sun $Y$, Ma X, et al. Androgen receptor increases hematogenous metastasis yet decreases lymphatic metastasis of renal cell carcinoma. Nat Commun 2017;8:918.

2. Sountoulides P, Metaxa L, Cindolo L. Atypical presentations and rare metastatic sites of renal cell carcinoma: a review of case reports. J Med Case Reports 2011;5:429.

3. Glazer AA, Novick AC. Long-Term Followup After Surgical Treatment for Renal Cell Carcinoma Extending into the Right Atrium. J Urol 1996;155(2):448-450.

4. Anis A, Maldjian P, Klapholz M, et al. M. Renal cell carcinoma with extension to the heart. Can J Cardiol 2008;24(11): 860.

5. Pala S, Erkol A, Kahveci G. Massive right atrial metastasis from renal cell carcinoma without inferior vena cava involvement. Turk Kardiyol Dern Ars 2009;37(5):358-358.

6. Marangoni G, O'Sullivan A, Ali A, et al. Budd-Chiari syndrome secondary to caval recurrence of renal cell carcinoma. Hepatobiliary Pancreat Dis Int 2010;9(3):321-324. 